\title{
Total Colorings of Planar Graphs with Large Maximum Degree
}

\author{
O. V. Borodin, ${ }^{1, *}$ A. V. Kostochka, ${ }^{2, \dagger}$ and D. R. Woodall ${ }^{3}$ \\ ${ }^{1}$ NOVOSIBIRSK STATE UNIVERSITY \\ NOVOSIBIRSK, 630090, RUSSIA \\ 2 INSTITUTE OF MATHEMATICS \\ SIBERIAN BRANCH, RUSSIAN ACADEMY OF SCIENCES \\ NOVOSIBIRSK, 630090, RUSSIA \\ ${ }^{3}$ DEPARTMENT OF MATHEMATICS \\ UNIVERSITY OF NOTTINGHAM \\ NOTTINGHAM, NG7 2RD, ENGLAND
}

Received April 29, 1996

\begin{abstract}
It is proved that a planar graph with maximum degree $\Delta \geq 11$ has total (vertex-edge) chromatic number $\Delta+1$. (C) 1997 John Wiley \& Sons, Inc. J Graph Theory 26: $53-59,1997$
\end{abstract}

Keywords: total coloring, planar graphs, discharging

\section{INTRODUCTION}

The total chromatic number $\chi^{\prime \prime}=\chi^{\prime \prime}(G)$ of a graph $G$ is the smallest number of colors that suffice to color the vertices and edges of $G$ so that no two adjacent or incident elements have the same color. It is clear that $\chi^{\prime \prime} \geq \Delta+1$, and Behzad [1] and Vizing [12] conjectured that

* This work was carried out while the first author was visiting Nottingham, funded by Visiting Fellowship Research Grant GR/K00561 from the Engineering and Physical Sciences Research Council.

$\dagger$ The work of the second author was partly supported by grant 96-01-01614 of the Russian Foundation for Fundamental Research and by the Program DIMANET/PECO of the European Union.

(c) 1997 John Wiley \& Sons, Inc.

CCC 0364-9024/97/010053-07 
$\chi^{\prime \prime} \leq \Delta+2$, for every graph $G$ with maximum degree $\Delta$. This conjecture was verified by Rosenfeld [10] and Vijayaditya [11] for $\Delta=3$ and by Kostochka [7, 8, 9] for $\Delta \leq 5$. For planar graphs the conjecture was verified by Borodin [2] for $\Delta \geq 9$, and now remains open only for $6 \leq \Delta \leq 7$ [6]. But for planar graphs it is often possible to obtain the exact result $\chi^{\prime \prime}=\Delta+1$. We proved this in [3] for planar graphs with various combinations of maximum degree and girth. With no condition on the girth, Borodin [2] proved it for planar graphs with maximum degree $\Delta \geq 14$. In [4] we improved this to $\Delta \geq 12$ (and, moreover, with the list total chromatic number $\chi_{\text {list }}^{\prime \prime}$ in place of $\left.\chi^{\prime \prime}\right)$. This paper is devoted to a proof of the following further improvement for $\chi^{\prime \prime}$.

Theorem. If $G$ is a planar graph with maximum degree $\Delta \geq 11$, then $\chi^{\prime \prime}(G)=\Delta+1$.

In fact, we prove that if $\Delta \geq 11$ then $\chi^{\prime \prime}(G) \leq \Delta+1$ for all planar graphs with maximum degree at most $\Delta$, and we let $G=(V, E)$ be a counterexample to this more general result with $|V|+|E|$ as small as possible, so that every proper subgraph of $G$ is totally $(\Delta+1)$-colorable. It is easy to see that $G$ is 2-connected, and hence has no vertices of degree 1.

\section{REDUCIBLE CONFIGURATIONS}

In this section we start the proof of the theorem by obtaining structural information about our minimal counterexample $G$, which shows that certain configurations are reducible, that is, they cannot occur in $G$. The degree of vertex $v$ is denoted by $d(v)$, and a $d$-vertex is a vertex $v$ with $d(v)=d$.

Lemma 1. (a) If $u w$ is an edge of $G$ with $d(u) \leq 5$, then $d(u)+d(w) \geq \Delta+2 \geq 13$ (and so 2 -vertices of $G$ are adjacent only to $\Delta$-vertices).

(b) The graph of all edges joining 2-vertices to $\Delta$-vertices is a forest.

Proof. If $G$ contains an edge $u w$ with $d(u) \leq 5$ and $d(u)+d(v) \leq \Delta+1$, then we can totally color $G-u w$ with $\Delta+1$ colors (by the minimality of $G$ ), erase the color on $u$, and then color $u w$ and $u$ in turn, since the number of colors that we may not use is at most $(\Delta-1)+1=\Delta$ for $u w$ and $5+5=10<\Delta$ for $u$. This contradicts the choice of $G$ as a counterexample and so proves (a).

If (b) is false then $G$ contains a cycle $v_{1} v_{2} \cdots v_{2 k} v_{1}$ of even length such that $d\left(v_{1}\right)=d\left(v_{3}\right)=$ $\cdots=d\left(v_{2 k-1}\right)=2$. Then, by the minimality of $G$, we can totally color $G-\left\{v_{1}, v_{3}, \ldots, v_{2 k-1}\right\}$ with $\Delta+1$ colors. Each edge of $C$ now has at most $(\Delta-2)+1=\Delta-1$ colors that may not be used on it, hence at least two that may, and so the problem of coloring the edges of $C$ is equivalent to coloring the vertices of an even cycle, given a choice of two colors at each vertex; it is well known $[5,13]$ that this is possible. The 2-vertices of $C$ are now easily colored, and this contradicts the choice of $G$ as a counterexample to the theorem, thereby proving (b).

In Figure 1, vertices marked $\bullet$ have no edges of $G$ incident with them other than those shown, and so have degree 2 or 3 in $G$.

Lemma 2. $G$ has no subgraph isomorphic to either of the configurations in Figure 1 (a) and (b).

Proof. Suppose that $G$ does contain one of these configurations. Choose a total coloring of $G-u v$ with $\Delta+1$ colors, which exists by the minimality of $G$, and erase the colors on the vertices marked $\bullet$. If we can prove that the resulting partial total coloring $c$ can be extended to edge $u v$, then we will have a contradiction to the choice of $G$ as a counterexample to the theorem, 


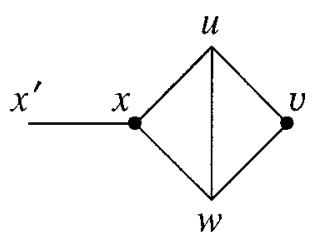

(a)

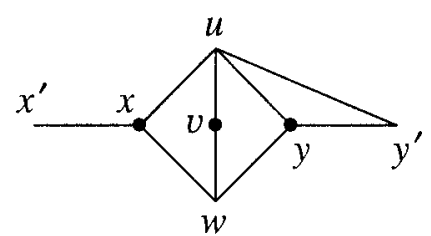

(b)

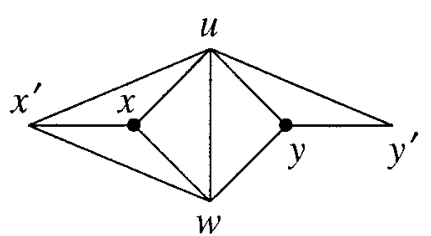

(c)

FIGURE 1. Some reducible configurations.

since vertices with degree at most 3 are easily colored. Let $c(v w)=1$. We may suppose that color 1 is not present at $u$ (that is, $1 \neq c(u)$ or $c(e)$ for any edge $e$ incident with $u$ ), since if it is then some other color is not present at $u$ and can be given to $u v$. The proof now divides.

(a) Since $1=c(v w)$ is not present at $u$, the three edges in the path vwux have three different colors, say 1, 2, 3 (in that order). Recolor these edges $1,2,1$ or $2,1,2$ according as $c\left(x x^{\prime}\right)=2$ or $\neq 2$, and color $u v$ with 3 .

(b) We may suppose that $c\left(x x^{\prime}\right)=c\left(y y^{\prime}\right)=1$, since otherwise we can give color $c(u x)$ or $c(u y)$ to $u v$ and recolor $u x$ or $u y$ with 1. Thus the edges of path $v w y y^{\prime} u$ have colors (w.l.o.g.) $1,2,1,3$ or $1,2,1,2$. In the former case, recolor these edges $1,2,3,1$ and color $u v$ with 3 . In the latter case, recolor the edges of path $v w y y^{\prime} u x$ with $2,1,2,1,2$ and give color $c(u x)$ to $u v$.

Lemma 3. $G$ has no subgraph isomorphic to the configuration in Figure 1(c).

Proof. Suppose it has. Let $C$ denote the 4-cycle $u x w y u$. Color $G-E(C)$ and erase the colors on $x, y$. Choose this coloring $c$ if possible so that $c\left(x x^{\prime}\right) \neq c\left(y y^{\prime}\right)$ and $c(u w) \in\left\{c\left(x x^{\prime}\right), c\left(y y^{\prime}\right)\right\}$. We now show that this is possible.

Suppose first that $c\left(x x^{\prime}\right)=c\left(y y^{\prime}\right),=1$, say. If color 1 is present at both $u$ and $w$ then there are at least two colors available for each edge of $C$, which leads to a contradiction since an even cycle is list-edge-2-colorable (and $x$ and $y$ are then easily colored). Thus color 1 is absent at $u$ or $w$, and we can ensure $c\left(x x^{\prime}\right) \neq c\left(y y^{\prime}\right)$ by swapping the color of $x x^{\prime}$ with that of $x^{\prime} u$ or $x^{\prime} w$ respectively. So from now on, let $c\left(x x^{\prime}\right)=1, c\left(y y^{\prime}\right)=2$.

Now suppose that $c(u w) \notin\{1,2\}$. If we cannot change $c(u w)$ to 1 or 2 then each of these colors is present at at least one of $u, w$. If both are present at $u$ (say), then two other colors are absent at $u$ and we can color $w x, w y$ first and then $u x, u y$. If however 1 (only) is present at $u$ and 2 (only) at $w$, then after coloring $u x, w y$ with 2,1 , there remains a color for each of $u y, w x$. This contradiction shows that we may suppose $c(u w) \in\{1,2\}$.

Let $O(v)$ denote the set of colors absent at vertex $v$. If $c(u w)=1$, it is now easy to complete the coloring unless $|O(u)|=|O(w)|=2$ and $2 \in O(u) \cap O(w)$, in which case we might have to give color 2 to both $u x$ and $w x$. The same applies with 1 and 2 interchanged. In either case, $|O(u) \backslash\{1,2\}|=|O(w) \backslash\{1,2\}|=1$, say $O(v) \backslash\{1,2\}=\{\bar{c}(v)\}(v=u, w)$. By symmetry (since we shall make no further use of $x^{\prime} w$ ) we may suppose that $c\left(u y^{\prime}\right)=3 \neq \bar{c}(w)$. Now we can color or recolor the edges of the trail wyy'uxwuy with colors 2, 3, 2, 3, $\bar{c}(w), 1, \bar{c}(u)$. Finally, $x$ and $y$ are easily colored.

Lemma 4. No face of $G$ has more than one 2-vertex in its boundary.

Proof. Suppose that $v, x$ are 2-vertices in the boundary of a face $f$. If $v, x$ are separated by at least two other vertices each way round the boundary, then we can simply identify them; the resulting simple planar graph is totally $(\Delta+1)$-colorable by the minimality of $G$, and this gives a 
total- $(\Delta+1)$-coloring of $G$. So suppose that $u, v, w, x, y$ are consecutive vertices in the boundary of $f$. By Lemma 1 (a) and (b), $u \neq y$. So identifying $v$ with $x$ and deleting one of the edges $v w$ and $w x$, say $v w$, gives a simple planar graph, which has a total- $(\Delta+1)$-coloring $c$ by the minimality of $G$. Apply this coloring to $G$. If $c(x y)$ is absent at $w$, give this color to edge $v w$; if not, give edge $v w$ color $c(w x)$ and recolor $w x$ with a color that is absent at $w$ (and so different from $c(x y)$ ), finally recoloring $x$ if necessary.

\section{DISCHARGING}

We shall complete the proof of the theorem by using discharging in order to obtain a contradiction. Let $(V, E, F)$ be a plane embedding of $G$. We assign a "charge" $M(\phi)$ to each element $\phi \in$ $V \cup F$, where

$$
M(\phi):= \begin{cases}d(\phi)-6 & \text { if } \phi \in V \\ 2 r(\phi)-6 & \text { if } \phi \in F\end{cases}
$$

where $r(f)$ denotes the number of edges around face $f$. Euler's formula $|V|-|E|+|F|=2$ can be rewritten in the form $(2|E|-6|V|)+(4|E|-6|F|)=-12$, which implies that

$$
\sum_{\phi \in V \cup F} M(\phi)=\sum_{v \in V}(d(v)-6)+\sum_{f \in F}(2 r(f)-6)=-12 .
$$

We shall now redistribute the charge, without changing its sum, in such a way that the sum is provably nonnegative, and this contradiction will prove the theorem. Before stating the redistribution rules, we need some definitions.

A 4-face uvxy is special if there is a 3-face $v x y$ (and hence $d(x)=2$ ). We shall suppose that the embedding of $G$ is chosen so as to minimize the number of special 4-faces. This implies that

for each special 4-face uvxy, the edge $v y$ separates two triangles,

since otherwise we could reduce the number of special 4-faces by moving edge $v y$ across the path $v x y$.

A lo-face of $G$ is a 3-face or a special 4-face; a hi-face is any other face, that is, a nonspecial 4-face or a $\geq 5$-face. A lo-triangle of $G$ is a 3 -face in the graph obtained by deleting all 2-vertices of $G$ and their incident edges; thus by Lemma 4 it is either a 3-face of $G$ having no vertices of degree 2, or a triangle uvy containing a 3-face $v x y$ and a special 4-face $u v x y$ with $d(x)=2$.

By Lemma 1 (a) and (b), it is possible to find a matching in $G$ that pairs off all the 2-vertices with some of the $\Delta$-vertices: in each component of the forest in Lemma $1(\mathrm{~b})$, choose a $\Delta$-vertex $v$, and match each 2-vertex $u$ with the $\Delta$-vertex $w$ adjacent to $u$ that is further from $v$. (Note that the endvertices of this forest are all $\Delta$-vertices.) We call $w$ the master of $u$ and $u$ the (unique) 2-dependant of $w$.

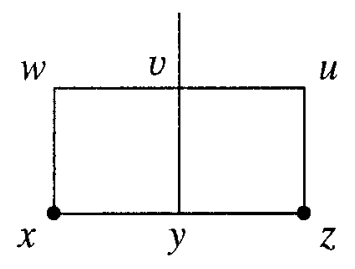

FIGURE 2. Vertex $v$ is a sponsor of vertex $y$. 
If the configuration in Figure 2 occurs in $G$, where $d(x)=d(z)=2, d(v) \geq 4$ and neither of the 4-faces vwxy and vuzy is special, then we say that $v$ is a sponsor of $y$. Note that $y$ in turn can sponsor other vertices, and even cycles of sponsorship are possible.

The rules for redistribution of charge are as follows.

R1: A special 4-face gives charge 2 to its 2-vertex. A nonspecial 4-face gives 1 to an incident $\leq 5$-vertex and 1 to the vertex opposite it. A $\geq 5$-face gives 3 to a 2 -vertex and 1 to any other $\leq 5$-vertex.

R2: Suppose $d(v)=2$. If $v$ is incident with a $\geq 5$-face, then $v$ receives 1 from its master. Otherwise, $v$ receives $13 / 10$ from its master and 7/10 from its other neighbor.

R3: Suppose $d(v)=3$. If $v$ is not incident with hi-faces, then $v$ receives 1 from each of its neighbors. If $v$ is incident with precisely one hi-face $\cdots a v b \cdots$ then $v$ receives $3 / 5$ from each of $a, b$ and $4 / 5$ from its third neighbor. If $v$ is incident with precisely two hi-faces $\cdots a v b \cdots$ and $\cdots b v c \cdots$ then $v$ receives $1 / 2$ from each of $a, c$.

R4: If $d(v)=4$ or 5 then $v$ receives $1 / 2$ along every incident edge that separates two lo-faces.

R5: A vertex gives $1 / 5$ to any vertex of which it is a sponsor.

R6: If $f=v x w y$ is a nonspecial 4-face with $d(x)=2$ and $d(y) \geq 10$, so that $f$ gives 1 to $y$ by $\mathrm{R} 1$, then $y$ gives $1 / 2$ to each of $v$ and $w$.

Let $M^{*}(\phi)$ be the resulting charge on $\phi$, so that $\sum_{\phi \in V \cup F} M^{*}(\phi)=-12$ by (1). We shall obtain a contradiction by proving that $M^{*}(\phi) \geq 0$ for each element $\phi$. We start with the easy cases.

Suppose first that $\phi$ is a face $f$. If $r(f)=3$ then $M^{*}(f)=M(f)=0$. If $r(f)=4$ then $M(f)=2$ and (by Lemma 1 (a)) $f$ gives up at most 2 by R1. If $r(f)=r \geq 5$ then $M(f)=2 r-6$ and (by Lemma 4) $f$ gives up at most $2+\left\lfloor\frac{1}{2} r\right\rfloor$ by R1. Thus $M^{*}(f) \geq 0$ in all cases.

Suppose now that $\phi$ is a vertex $v$. If $d(v)=2$ then $M(v)=-4$. If $v$ is incident with a $\geq 5$-face $f$ then $v$ receives 3 from $f$ by $\mathrm{R} 1$ and 1 from its master by $\mathrm{R} 2$. The only other possibilities (since $G$ is simple) are that $v$ is between two (nonspecial) 4-faces, or between a (special) 4-face and a 3 -face, in which case $v$ receives 2 from the 4 -face(s) by $\mathrm{R} 1$ and 2 from neighboring vertices by R2. In either case, $M^{*}(v)=0$.

If $d(v)=3$ then $M(v)=-3$. By $\mathrm{R} 1, v$ receives 1 from any incident hi-face, and it is easy to see from R3 that $M^{*}(v)=0$.

If $d(v)=4$ or 5 then $M(v)=-2$ or -1 . By R1, $v$ receives 1 from any incident hi-face. By this and $\mathrm{R} 4$, it is easy to see that $M^{*}(v) \geq 0$ if $v$ is not a sponsor. If $v$ is a sponsor, then it receives 2 from the two hi-faces (non-special 4-faces) involved in the sponsorship. If there is another hi-face at $v$ then $v$ receives another 1 which suffices for five sponsorships; otherwise, the other faces are all lo-faces and $v$ receives at least $\frac{1}{2}$ between them, which covers its one sponsorship.

If $d(v)=6$ or 7 then $M(v) \geq 0$ and $v$ gives nothing if it is not a sponsor. If $v$ is a sponsor then it receives 2 from the two hi-faces (non-special 4-faces) involved in the sponsorship, and this suffices for $10>7$ sponsorships.

If $d(v)=8$ or 9 then $M(v) \geq 2$ and $v$ can give $\frac{1}{2}$ to at most four vertices by R4. If $v$ is a sponsor then it receives an additional 2 from two hi-faces, which suffices for $10>9$ sponsorships.

If $d(v) \geq 10$ then, in addition to giving charge by $\mathrm{R} 4$ and $\mathrm{R} 5, v$ can give charge to 3-vertices by $\mathrm{R} 3$ and (if $d(v) \geq 11$ ) to 2 -vertices by $\mathrm{R} 2$. We shall deal with this case in the next section.

\section{VERTICES WITH DEGREE AT LEAST 10}

Let $v$ be a fixed vertex with $d(v) \geq 10$. For the sole purpose of proving $M^{*}(v) \geq 0$, we carry out a temporary local redistribution of charge whereby some neighbors of $v$ that have received 
charge from $v$ will give some of it to other neighbors of $v$ that have received no charge from $v$. We shall prove that, after this local redistribution,

$v$ gives 1 to its 2-dependant (if any) and at most $\frac{2}{5}$ to each other neighbor.

This will suffice to prove the result, since it shows that $M^{*}(v) \geq(d(v)-6)-1-\frac{2}{5}(d(v)-1)=$ $\frac{3}{5}(d(v)-11) \geq 0$ if $d(v) \geq 11$, while if $d(v)=10$ then $v$ is not adjacent to any 2-vertices by Lemma 1(a) and so $M^{*}(v) \geq(d(v)-6)-\frac{2}{5} d(v)=0$.

Let $x$ be a neighbor of $v$ that has received "too much" charge from $v$ (that is, more than is allowed for in (3)). The following rules for the temporary local redistribution of charge cover all possibilities for $x$. (Note that $v$ cannot be a sponsor of $x$ if $d(x)<\Delta$, by Lemma 1 (a) and the structure of Figure 2.)

L1: Suppose that $x$ is a 2-vertex that has received $\frac{13}{10}$ or $\frac{7}{10}$ from $v$ by R2. If edge $v x$ separates a 3-face $v x y$ from a special 4-face $u v x y$, then $x$ now gives $\frac{3}{10}$ to $y$ (and so retains 1 or $\frac{2}{5}$ ). The only other possibility is that $v x$ separates two nonspecial 4-faces uvxy and wvxy, in which case $x$ now gives $\frac{3}{20}$ to each of $u, w$ unless one has degree 3 and the other has larger degree, in which case $x$ gives $\frac{3}{10}$ to whichever has larger degree. (The reason for this is that a 3-vertex could have received charge from $v$ by R3. However, this cannot happen if both $u$ and $w$ have degree 3, since then neither belongs to a triangle by Lemma 2(b).)

L2: Suppose $d(x)=3$. Suppose first that $v x$ separates two lo-triangles $v x y_{1}$ and $v x y_{2}$. If $x y_{1} y_{2}$ is a lo-triangle then $x$ has received 1 from $v$ by R3 and now gives $\frac{3}{10}$ to each of $y_{1}, y_{2}$; otherwise $x$ has received $\frac{4}{5}$ from $v$ by R3 and now gives $\frac{1}{5}$ to each of $y_{1}, y_{2}$. Suppose now that $v x$ separates a lo-triangle $v x y$ from a hi-face. If there is another lo-triangle at $x$ then $x$ has received $\frac{3}{5}$ from $v$ by R 3 and now gives $\frac{1}{5}$ to $y$; otherwise $x$ has received $\frac{1}{2}$ from $v$ by R3 and now gives $\frac{1}{10}$ to $y$. (If $v x$ separates two hi-faces then $x$ has received nothing from $v$.)

L3: Suppose that $d(x)=4$ or 5 and $x$ has received $\frac{1}{2}$ from $v$ by R4. Then $v x$ separates two lo-triangles $v x y_{1}$ and $v x y_{2}$, and $x$ now gives $\frac{1}{20}$ to each of $y_{1}, y_{2}$.

Note that the redistribution in L2 and L3 always happens along an edge $x y$ of a triangle $v x y$.

It is clear from L1-L3 that (3) holds for any neighbor of $v$ that received charge from $v$ by R1-R6, since no such neighbor receives anything more by L1-L3. So let $y$ be a neighbor of $v$ that receives charge by L1-L3. We must prove that it receives at most $\frac{2}{5}$.

Suppose first that $y$ receives $\frac{3}{10}$ by L1 from a 2-vertex $x$ adjacent to both $v$ and $y$. Then the path $v x y$ separates a 3-face $v x y$ from a special 4-face $u v x y$, and by (2) the edge $v y$ separates two 3-faces, say $v x y$ and $v w y$. By Lemma 2 (a), $d(u) \geq 4$ and $d(w) \geq 4$. By L3, $y$ receives at most $\frac{1}{20}$ from each of $u, w$, and nothing from any other vertex except $x$, making a total of at most $\frac{3}{10}+\frac{2}{20}=\frac{2}{5}$.

Suppose now that $y$ receives $\frac{3}{10}$ or $\frac{3}{20}$ from a 2-vertex $x$ across a 4-face by L1, so that $v x$ lies between nonspecial 4-faces $v x w y$ and $v x w z$, say. If $d(y)=3$ then $y$ receives $\frac{3}{20}$ from $x$ and at most $\frac{3}{20}$ from a 2 -vertex on the other side of $v y$, making less than $\frac{2}{5}$ in total. If $4 \leq d(y) \leq 9$ then $y$ is not adjacent to 3 -vertices by Lemma 1(a); it receives at most $\frac{3}{10}$ from $x$ and nothing more unless it receives $\frac{3}{10}$ from a 2-vertex on the other side of $v y$, in which case $y$ is a sponsor of $v$ and gives back $\frac{1}{5}$ to $v$ by $\mathrm{R} 5$, thus retaining exactly $\frac{2}{5}$ from $v$. If $d(y) \geq 10$ then $y$ can receive up to $\frac{3}{10}$ from $x$ and $\frac{3}{10}$ from a 2-vertex or 3-vertex on the other side of $v y$, but $y$ gives $\frac{1}{2}$ to $v$ by R6, and so retains at most $\frac{1}{10}$ from $v$.

Finally, suppose $y$ receives nothing from 2-vertices. If $y$ is sponsored by $v$ then it receives $\frac{1}{5}$ and nothing more from $v$. Assume $y$ is not sponsored by $v$. By L2 and L3, $y$ can get 
only $\frac{3}{10}, \frac{1}{5}, \frac{1}{10}$ or $\frac{1}{20}$ from at most two $\leq 5$-vertices $x_{1}, x_{2}$ lying in triangles $v y x_{1}$ and $v y x_{2}$. To receive more than $\frac{2}{5}$ in total, $y$ must receive $\frac{3}{10}$ from $x_{1}$ or $x_{2}$, say $x_{1}$, and either $\frac{3}{10}$ or $\frac{1}{5}$ from $x_{2}$. By L2, there are three lo-triangles at $x_{1}$ and two or three lo-triangles at $x_{2}$. But this contradicts Lemma 3, and this contradiction completes the proof of the Theorem.

\section{References}

[1] M. Behzad, Graphs and their chromatic numbers, Doctoral Thesis, Michigan State University (1965).

[2] O. V. Borodin, On the total colouring of planar graphs, J. Reine Angew. Math. 394 (1989), 180-185.

[3] O. V. Borodin, A. V. Kostochka, and D. R. Woodall, Total colourings of planar graphs with large girth, submitted.

[4] O. V. Borodin, A. V. Kostochka, and D. R. Woodall, List edge and list total colourings of multigraphs, submitted.

[5] P. Erdős, A. L. Rubin, and H. Taylor, Choosability in graphs, in Proc. West Coast Conference on Combinatorics, Graph Theory and Computing, Arcata, 1979, Congr. Numer. 26 (1979), 125-157.

[6] T. R. Jensen and B. Toft, Graph Coloring Problems, Wiley, New York (1995), 88.

[7] A. V. Kostochka, The total coloring of a multigraph with maximal degree 4, Discrete Math. 17 (1977), $161-163$.

[8] A. V. Kostochka, Upper bounds of chromatic functions on graphs (in Russian), Doctoral Thesis, Novosibirsk (1978).

[9] A. V. Kostochka, The total chromatic number of any multigraph with maximum degree five is at most seven, Discrete Math. 162 (1996), 199-214.

[10] M. Rosenfeld, On the total coloring of certain graphs, Israel J. Math. 9 (1971), 396-402.

[11] N. Vijayaditya, On total chromatic number of a graph, J. London Math. Soc. (2) 3 (1971), 405-408.

[12] V. G. Vizing, Some unsolved problems in graph theory (in Russian), Uspekhi Mat. Nauk 23 (1968), 117-134. English translation in Russian Math. Surveys 23, 125-141.

[13] V. G. Vizing, Vertex colouring with given colours (in Russian), Metody Diskret. Analiz. 29 (1976), $3-10$. 